\title{
Improvements on the performance of subcarrier multiplexing/wavelength division multiplexing based radio over fiber system
}

\author{
Duc-Tan Tran ${ }^{1}$, Ninh Trung Bui ${ }^{2}$ \\ ${ }^{1}$ Faculty of Electrical and Electronic Engineering, Phenikaa University, Hanoi, Vietnam \\ ${ }^{1}$ Phenikaa Research and Technology Institute (PRATI), A\&A Green Phoenix Group JSC, Hanoi, Vietnam \\ ${ }^{2}$ VNU University of Engineering and Technology (VNU-UET), Vietnam National University, Hanoi, Vietnam
}

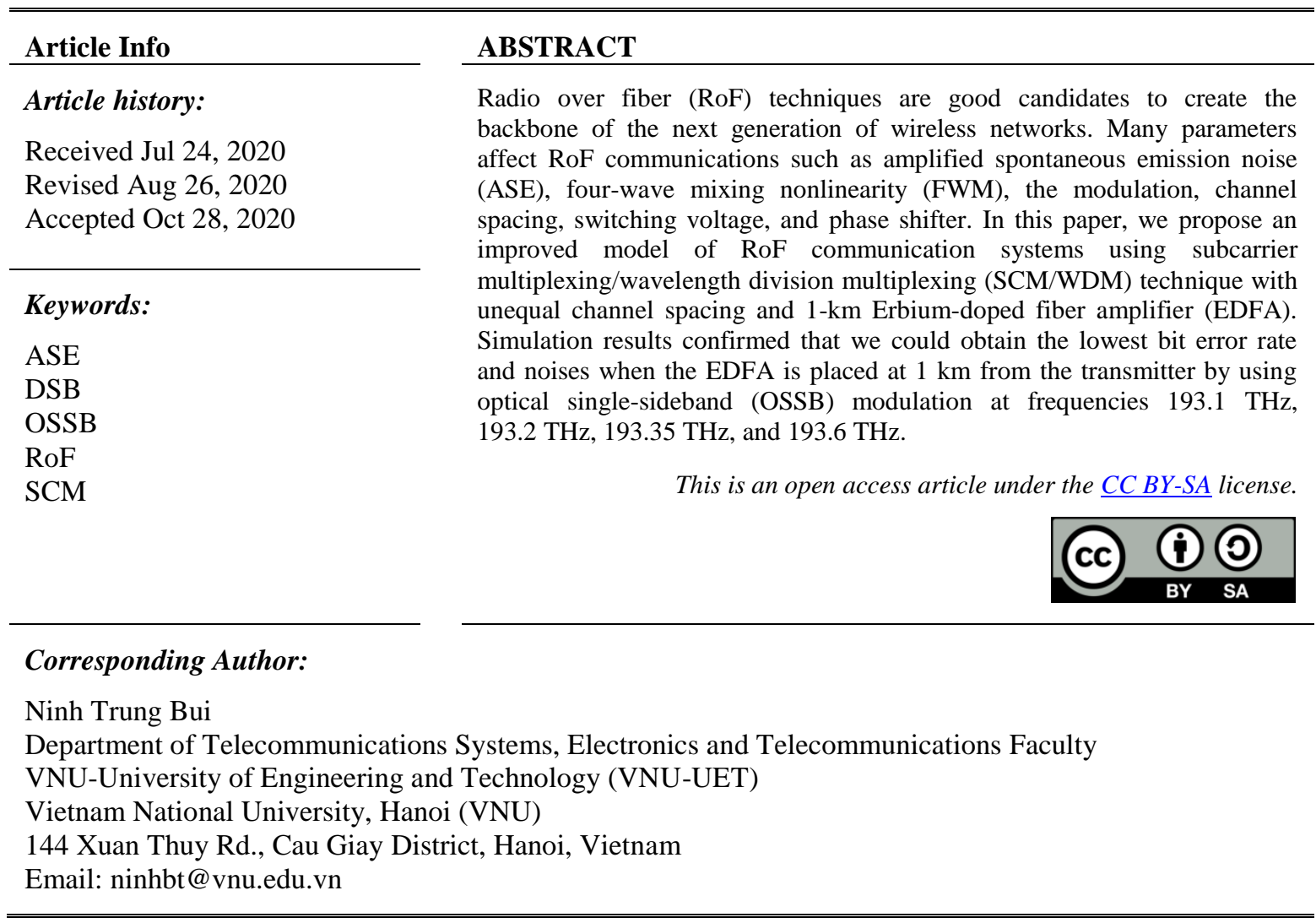

\section{INTRODUCTION}

Radio over fiber (RoF) is one of the solutions for modern communication systems. It is used to combine optical fiber communication and wireless mobile networks. This combination provides high-quality signals, low cost, and high bandwidth, which brings many advantages to conventional wireless communication systems [1-4]. RoF allows modulation of the optical source by electrical signals, and then the optical signal will be transmitted via optical fiber to the remote station [5]. RoF allows reducing power consumption while the remoted side has high-frequency carriers.

Wavelength division multiplexing (WDM) is a technique that allows multiplexing many wavelengths and transmission in a single optical fiber. It helps increase the bit rate, capacity, flexibility, and reduce the cost of optical communication systems $[6,7]$. WDM is also used in the RoF system to increase the capacity and bandwidth of wireless systems. This combination significantly increases coverage area and mobility of optical and wireless mobile networks. However, the performance of WDM-RoF systems is severely affected by the nonlinear effect in fiber optics, especially FWM [8]. To reduce the influence of FWM, we can use the mechanism of channel allocating with unequal spacing [9], the pairing combinations of differently linear-polarized optical signals [10]. 
Optical subcarrier multiplexing (SCM) is a technique that allows multiple signals are multiplexed in the frequency domain and then transmitted on optical fiber $[11,12]$. The signals are individually modulated with different frequencies. They are added together by an RF (radio frequency) multiplexer before converting into an optical signal through an optical source and optical modulator [13]. SCM can carry many closed different optical carriers. Therefore, the bandwidth utilization efficiency will be higher than in the traditional WDM system [14].

When the RF signal is transferred to the optical domain using an optical modulator, two side-bands will be created in the frequency domain. This double side-band (DSB) signal is affected by the chromatic dispersion [15-17], the fiber length, the fiber dispersion parameter, the wavelength, and the modulation frequency, which creates a phase difference between the two bands to reduce system performance. To overcome this phenomenon, we can use some techniques such as: to use dispersion-compensating optical fibers [18], an optical filter (Bragg grating filter) to eliminate one side-band, or using advanced modulations to achieve an optical single-sideband (OSSB) signal.

There have been some previous works surveyed about the impact of switching voltage of MachZehnder modulator on SCM/ASK RoF system [19, 20]. Still, only one wavelength has been analyzed, and the optimal value of switching voltage has not been determined according to DSB or OSSB. The values of the optimal switching voltage and BER are high $\left(8 \mathrm{~V}, 10^{-16}\right.$, or $\left.10^{-17}\right)$. Articles [21-26] analyze SCM/WDM RoF systems' performance using OSSB modulation but have not yet compared its BER with the DSB modulation technique. Besides, these articles did not mention the effects of ASE noise when using EDFA amplifiers as well as the effects of FWM nonlinear effects and channel spacing in SCM/WDM RoF systems. In [27], the authors evaluated the performance of the four-channel WDM RoF-EPON link based on OVSB using SOA, DCF, and FBG. They used unequal channel spacing but have not investigated the effect of ASE noise and have not found the best location to place the amplifier.

In this paper, we firstly analyze RoF systems using SCM/WDM architecture with equal channel spacing (four optical carriers at frequencies 193.1, 193.2, 193.3, and 193.4 THz). Secondly, we proposed to use unequal channel spacing (four optical carriers at frequencies 193.1, 193.2, 193.35, and 193.6 THz) to transmit 78 analog channels and one digital amplitude-shift keying (ASK) signal at frequencies 1.5, 2, 2.5, and $3 \mathrm{GHz}$, respectively. We also investigate the effects of ASE noise, FWM, and the amplifier's location to bit error rate of the system. We found that if the EDFA is placed at $1 \mathrm{~km}$ from the transmitter, the ASE noise would be eliminated. Finally, our improved model of RoF communication system is compared to the conventional one in terms of BER.

The remainder of this article is organized as follows. In Section 2, we indicate the theoretical analysis of the SCM/WDM RoF system. In Section 3, we present the system's simulation setup, the achieved results, and discussion. Finally, Section 4 concludes the paper.

\section{THEORETICAL ANALYSIS}

In this section, we will discuss the OSSB technique and characteristics of optical fiber such as attenuation, dispersion, FWM, and noise.

\subsection{SCM uses optical single-sideband (OSSB)}

Figure 1 shows the single-sideband modulation structure using a dual-drive Mach-Zehnder modulator (MZM) [28, 29]. The modulating and carrier signals are fed into two branches of dual-driver MZM at $90^{\circ}$ out of phase. The modulated signal is then combined by an adder/subtracts to create single sideband modulation.

The output signal of the modulator is:

$$
s(t)=A_{c} A_{0} \operatorname{Cos}\left(\omega_{0}-\omega_{c}\right) t \text { or } A_{c} A_{0} \operatorname{Cos}\left(\omega_{0}+\omega_{c}\right) t
$$

where $A_{c}, A_{0}, \omega_{c}, \omega_{0}$ are the amplitudes and the angular frequencies $(\omega=2 \pi f)$ of the RF signal and the optical wave, respectively.

The corresponding spectrum of OSSB signal:

$$
\begin{aligned}
& s(f)_{\text {Low } B}=\frac{A_{c} A_{0}}{2}\left\{S\left[\omega-\left(\omega_{0}-\omega_{c}\right)\right]+S\left[\omega+\left(\omega_{0}-\omega_{c}\right)\right]\right\} \\
& s(f)_{U p S B}=\frac{A_{c} A_{0}}{2}\left\{S\left[\omega-\left(\omega_{0}+\omega_{c}\right)\right]+S\left[\omega+\left(\omega_{0}+\omega_{c}\right)\right]\right\}
\end{aligned}
$$

The optical power at the output of the dual driver MZM equals to: 


$$
P_{\text {dual }}(t)=\left|E_{\text {dual }}(t)\right|^{2}=P_{\text {in }}\left[1+\cos \left(\frac{\pi V_{\text {bias }}}{V_{\pi}}+\frac{\pi V(t)}{V_{\pi}}\right)\right]
$$

where $E_{\text {dual }}(t)$ is the optical field at the output of the MZM modulator, and $P_{\text {in }}$ is the input optical power.

The photocurrent at the output of the photodetector for modulation case at the quadrature point (QP) with $V_{\text {bias }}=V_{\pi} / 2+m 2 V_{\pi}$ or $V_{\text {bias }}=-V_{\pi} / 2+m 2 V_{\pi}$ is:

$$
\begin{aligned}
& I_{Q P}(t) \sim P_{Q P}(t)=P_{\text {in }}\left[\mp \sin \sin \left(\frac{\pi V_{L O} \cos \cos \left(\omega_{L O} t\right)}{V_{\pi}}\right)\right] \\
& =P_{\text {in }}\left[\frac{1}{2} \pm \sum_{n=1}^{\infty} \quad(-1)^{n} J_{2 n-1}\left(\frac{\pi V_{L O}}{V_{\pi}}\right) \times\right. \\
& \left.\quad \times \cos \left((2 n-1) \omega_{L O} t\right)\right],
\end{aligned}
$$

where $V(t)=V_{L O} \cos \cos \left(\omega_{L O} t\right)$ is a sine-modulated signal, and $J$ is a Jacobian function.

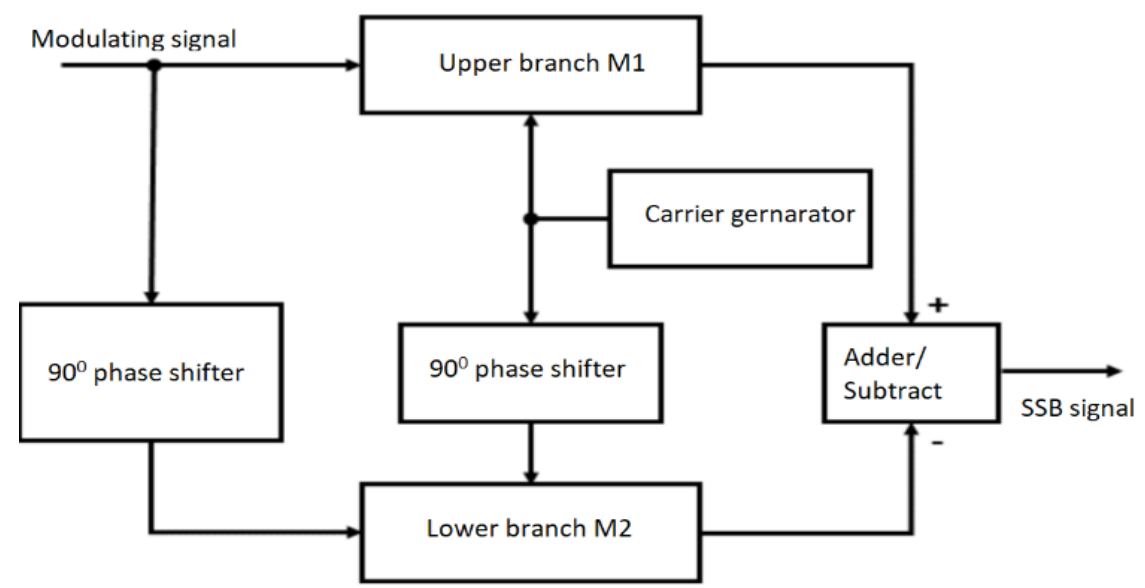

Figure 1. The structure of OSSB modulator

\subsection{The four-wave mixing effect in optical fiber}

FWM is a nonlinear effect in an optical fiber that occurs when the wavelengths are coupled tightly together in the WDM system. It is independent of bit rate but depends on channel spacing and chromatic dispersion of the fiber [30]. Let $\mathrm{N}$ refers to the number of wavelengths, $\mathrm{M}$ mixing products created in the optical fiber can be expressed as:

$$
M=\frac{N^{2}}{2}(N-1)
$$

Assuming there are three input wavelengths Figure 2(a): $\omega_{1,2,3}=193.1 \mathrm{THz}, 193.2 \mathrm{THz}$, and 193.3 THz (equal channel spacing). Then the mixing wavelengths generated beside the original wavelengths are $\omega_{1}+\omega_{2}-\omega_{3}=193 \mathrm{THz}, \omega_{1}-\omega_{2}+\omega_{3}=193.2 \mathrm{THz}, \omega_{2}+\omega_{3}-\omega_{1}=193.4 \mathrm{THz}, 2 \omega_{1}-\omega_{2}=$ $193 \mathrm{THz}, 2 \omega_{1}-\omega_{3}=192.9 \mathrm{THz}, 2 \omega_{2}-\omega_{1}=193.3 \mathrm{THz}, 2 \omega_{2}-\omega_{3}=193.1 \mathrm{THz}, 2 \omega_{3}-\omega_{1}=$ 193.5 THz, and $2 \omega_{3}-\omega_{2}=193.4 \mathrm{THz}$. When the channel spacing is not equal $(193.1 \mathrm{THz}, 193.2 \mathrm{THz}$, and $193.6 \mathrm{THz}$ ), the result is shown in Figure 2(b).

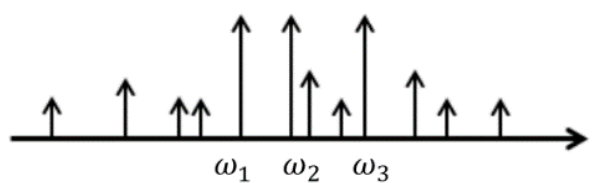

(a)

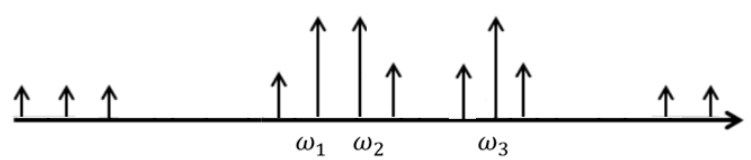

(b)

Figure 2. The arising side-bands due to FWM (three input wavelengths), (a) Equal channel spacing, (b) Unequal channel spacing 


\subsection{Attenuation and dispersion in the optical fiber}

The signal power will decrease when transmitted through the optical fiber due to the impurity of materials and Rayleigh scattering. Let $\alpha$ refers to the fiber attenuation coefficient in $\mathrm{km}-1$, and the receiver signal power depends on the transmitted power according to:

$$
P_{r}=P_{t} e^{-\alpha L}
$$

where $L$ is the optical fiber length, with SMF fibers, $\alpha_{d B}=0.2 \mathrm{~dB} / \mathrm{km}$.

The refractive index of a fiber depends on the frequency $f=\omega / 2 \pi$ of the optical wave, the phase velocity of light $v_{p}(\omega)=c / n(\omega)$ depends on the optical frequency that causes dispersion. The degree of dispersion is characterized by the dispersion parameter $\mathrm{D}$ as following:

$$
D=\frac{-2 \pi c}{\lambda_{0}^{2}} \beta_{2}
$$

The factor $\beta_{2}$ is called the group velocity dispersion parameter (GVD).

\subsection{Noises}

Noise components in the RoF system: shot noise, thermal noise, relative intensity noise (RIN) of the optical source, amplified spontaneous emission (ASE) noise caused by the amplifier [30, 31]. First, the variance of the shot noise, which is generated by the photon stream, is given by

$$
\sigma_{\text {shot }}^{2}=2 \mathrm{qI}_{\mathrm{dc}} \Delta \mathrm{f}
$$

where $q$ is the electron charge, $I_{d c}$ is photocurrent, $\Delta f$ is the bandwidth of the receiver.

Next, the variance of the thermal noise can be written as

$$
\sigma_{t h}^{2}=\frac{4 k_{B} T}{R_{L}} \Delta f
$$

where $k_{B}$ is Boltzman's constant, $\mathrm{T}$ is the receiver temperature, and $R_{L}$ is the load resistance.

The last one is the beat noise current. It consists of the signal-ASE beat noise, the ASE-ASE beat noise, and signal-signal beat noise. The total variance of the noise current is the sum of all variances of thermal noise, shot noise and beat noise and can be calculated by

$$
\sigma_{\text {total }}^{2}=\sigma_{\text {th }}^{2}+\sigma_{\text {sh }}^{2}+\sigma_{\text {beat }}^{2}
$$

Eventually, the bit error rate (BER) can be written as

$$
B E R=\frac{1}{2} \operatorname{erfc}(S N R)
$$

where $\operatorname{erfc(.)}$ is the complementary error function, and signal-to-noise rate is calculated by [30]

$$
\operatorname{SNR}=\frac{\mathrm{I}_{\text {signal }}(\mathrm{ith})}{\sigma_{\text {total }} \sqrt{2}}
$$

$I_{\text {signal }}(i t h)$ is a photocurrent of the $\mathrm{i}^{\text {th }}$ channel at the exit of the photodiode.

\section{SIMULATION AND EXPERIMENT RESULTS}

In this section, we propose a system model, present the results obtained, discuss, and compare with the previous works.

\subsection{Simulation setup}

In this section, we set up an SCM/WDM-based RoF system model by using OptiSystem 17 software to investigate the effect of ASE noise and modulation techniques on the system performance as shown in Figure 3. We used four transmitters in this model, each carrying 78 analog channels and one digital ASK signal. Laser sources emit continuous wave with frequencies $193.1 \mathrm{THz}, 193.2 \mathrm{THz}, 193.3 \mathrm{THz}$, and 193.4 THz, respectively, is modulated by the MZMs under the support of phase shifter (to create a single 
side-band signal-SSB) and local oscillators with frequencies of $10 \mathrm{GHz}, 20 \mathrm{GHz}, 30 \mathrm{GHz}$ and $40 \mathrm{GHz}$, respectively. The WDM multiplexer is used to multiplex signals from 4 transmitters. An EDFA amplifier with a gain of $10 \mathrm{~dB}$ is used to increase the power of the optical signal. The signal is then transmitted via single-mode fiber (SMF). On the receiver side, the demultiplexer is used for wavelengths filtering before it is distributed to the individual receivers. The signal is then converted into photocurrent by using a PIN photodetector. BER of the received signal is observed using a BER analyzer combined with a low pass Bessel filter.

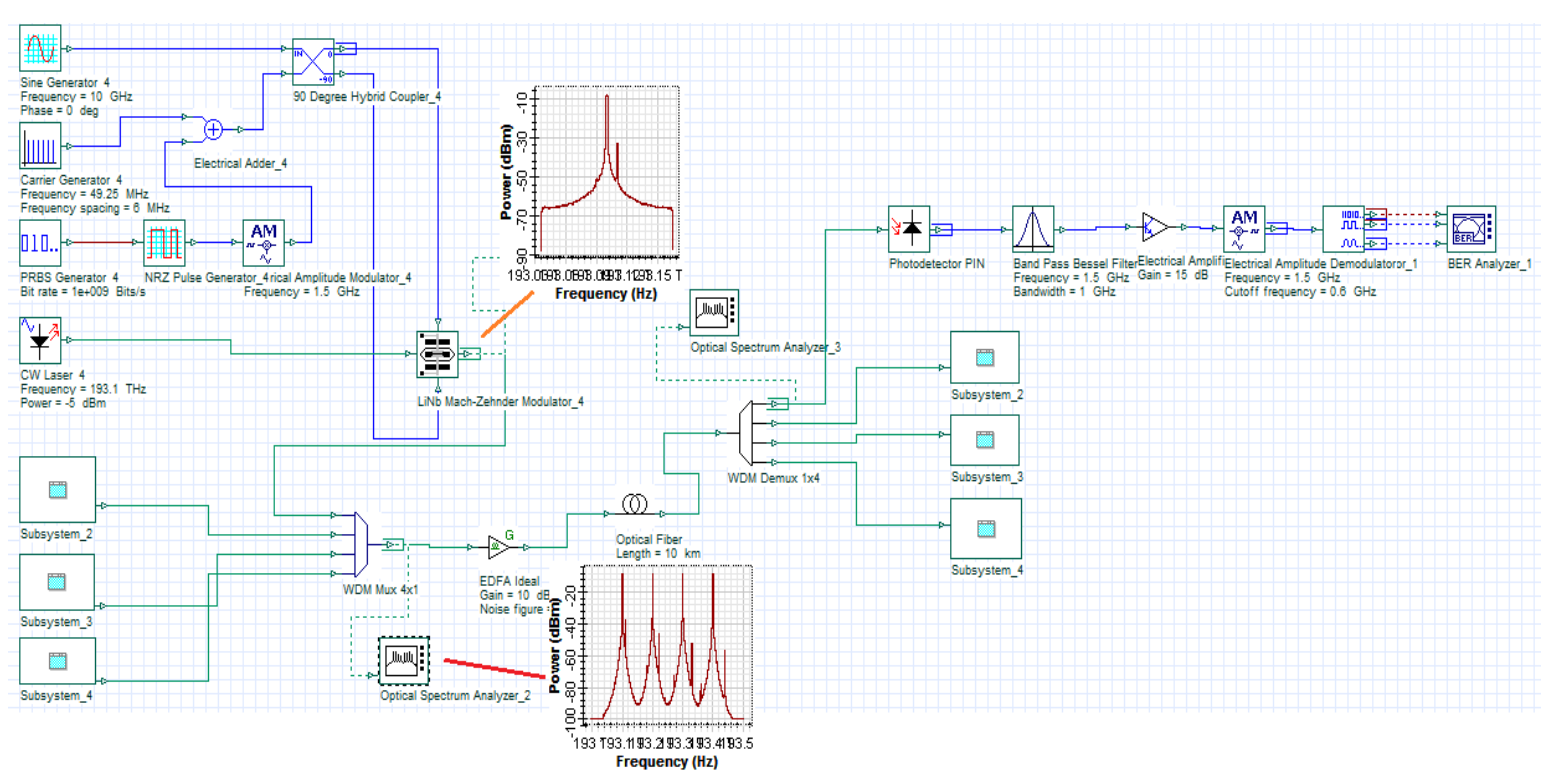

Figure 3. Block diagram of an RoF system using SCM/WDM

\subsection{Simulation results}

Simulations have been performed to consider the effects of ASE noise, switching voltage, bias voltage, and modulation method (DSB or OSSB) on the system's performance in different scenarios. The key parameters used for this simulation are listed in Table 1.

Table 1. Simulation parameters

\begin{tabular}{ccc}
\hline Name & Symbol & Value \\
\hline Length of optical fiber & $\mathrm{L}$ & $10 \mathrm{~km}$ \\
Effective area & $A_{\text {eff }}$ & $80 \mu^{2}$ \\
Bit rate & $R_{b}$ & $1 \mathrm{Gbps}$ \\
Optical frequencies & $f_{o}$ & $193.1-193.6 \mathrm{THz}$ \\
Carrier frequencies & $f_{c}$ & $1.5,2,2.5,3 \mathrm{GHz}$ \\
Attenuation coefficient & $\alpha_{d B}$ & $0.2 \mathrm{~dB} / \mathrm{km}$ \\
Dispersion & $\mathrm{D}$ & $16.75 \mathrm{ps} / \mathrm{nm} . \mathrm{km}$ \\
\hline
\end{tabular}

Figure 4 shows the relationship between BER and the switching bias voltage of the MZM modulation for two cases, with and without ASE noise. We fix the bias voltage at values of $V_{1}=0$ and $V_{2}=3$ volts and set the switching voltage to vary from 4 to 8.5 volts. It is seen that the values of the switching bias voltage at which the lowest BER can be achieved are from $5.5 \mathrm{~V}$ to $6 \mathrm{~V}$ (equal to $V_{\pi}$ ). In the absence of ASE, BER is very good. However, when ASE noise is considered, the BER increases significantly, and the most affected case is the RF signal channel of $1.5 \mathrm{GHz}$.

Figure 5 shows the BER as a function of the bias voltage 2. As we can see, when the bias voltage is $3 \mathrm{~V}=\frac{1}{2} V_{\pi}$ (the quadrature point at which the OSSB signal is generated). The BER is the lowest. Explicitly, the BER performance of a system in the case without ASE is much better than with ASE-case. 


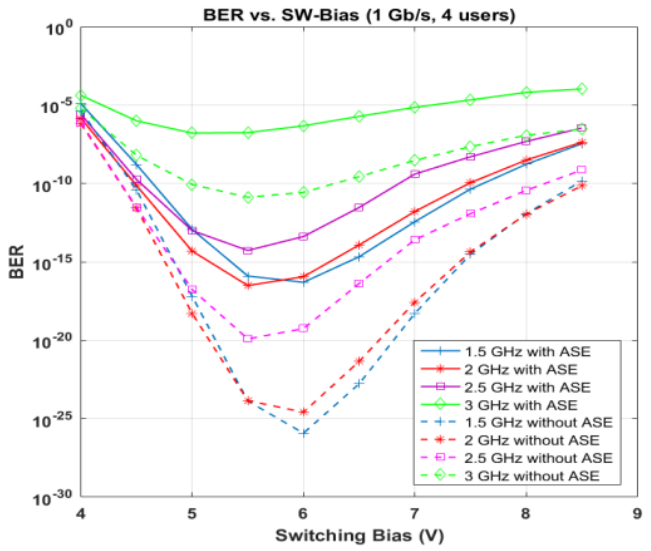

Figure 4. BER as a function of the switching voltage with $P_{\text {in }}=-5 \mathrm{dBm}$

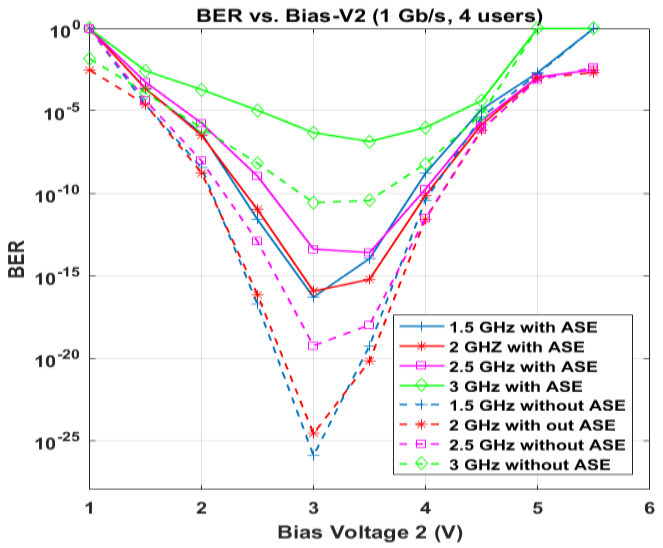

Figure 5. BER as a function of bias voltage 2 with $P_{\text {in }}=-5 \mathrm{dBm}$ and switching voltage of $6 \mathrm{~V}$

In Figure 6, we fix the transmitted power $P_{i n}=-5 \mathrm{dBm}$ and EDFA gain $=10 \mathrm{~dB}$. We examine BER versus extinction rate (Ex) for cases, with and without ASE noise, DSB, and SSB. It is realized that when Ex increases, BER is reduced. However, when Ex is greater than $15 \mathrm{~dB}$, BER curves will be saturated. It proves that the BER values will not change when Ex reaches a certain threshold. In the case of OSSB modulation, the BER is much better than the DSB modulation.

Figure 7 shows noise powers as a function of fiber length of the first $(1.5 \mathrm{GHz})$ and the fourth ( $3 \mathrm{GHz}$ ) RF channels when transmitted power $P_{i n}=-5 \mathrm{dBm}$ for two cases, DSB, and OSSB modulation. We can see, when fiber length increases, noise powers are decreased, and in the OSSB modulation case, noise powers of both the first and the fourth channels are smaller than those in DSB modulation. OSSB modulation is advantageous than DSB modulation from the viewpoint of minimizing noise. It is because the combination of the MZM modulator and the $90^{\circ}$ the phase shifter has eliminated a side-band of the modulated signal, which helps reduce the effects of noise and dispersion. This result is consistent with the theoretical analysis in section 2.1 .

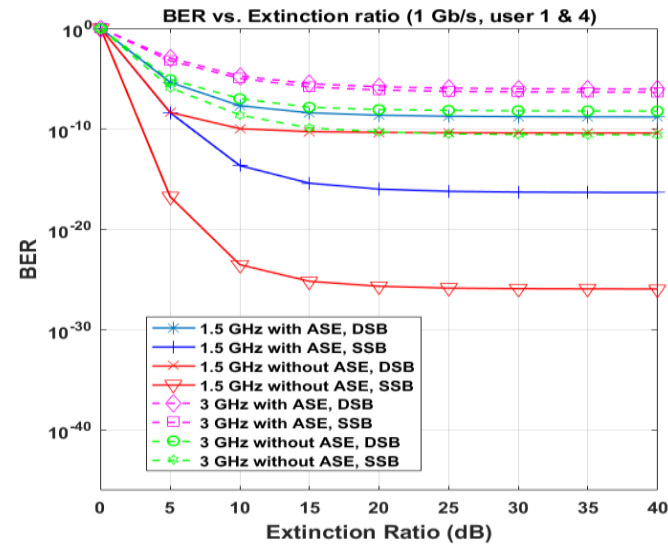

Figure 6. BER as a function of extinction rate $(\mathrm{Ex})$ with $P_{\text {in }}=-5 \mathrm{dBm}$

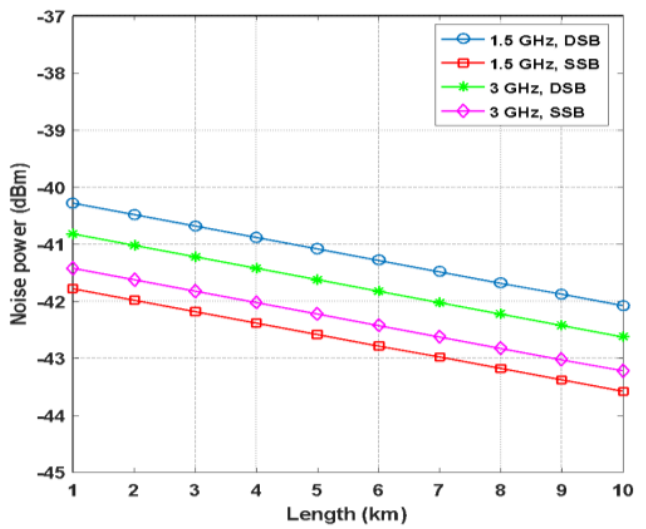

Figure 7. Noise power as a function of fiber length

From the results of Figures 4 and 5, we fix the switching voltage to $6 \mathrm{~V}$ and bias voltage to $3 \mathrm{~V}$, amplification factor $\mathrm{G}=10 \mathrm{~dB}$, and the transmission length of $10 \mathrm{~km}$. We consider BER versus transmitted power for two cases, with and without ASE noise in the DSB and OSSB modulation configurations for RF signal channel $1(1.5 \mathrm{GHz})$ and $4(3 \mathrm{GHz})$ as shown in Figure 8. It is seen that when transmitted power increases, the BER decreases. Moreover, the effect of ASE increases in DSB modulation case compared with OSSB modulation, and the effect of ASE noise in channel 1 is also higher than on channel 4. These results are consistent with the theoretical analysis in sections 2.1 and 2.4. 
Figures 9 and 10 shows BER's dependence on the transmitted power for two cases: DSB and OSSB modulations, with and without ASE. However, when the transmitted power is greater than $0 \mathrm{dBm}$, BER does not decrease any more; it falls into a saturated state. The reason is that the nonlinear effects in the optical fiber increase as the power increases. Moreover, in Figure 10, the OSSB modulation case, the BER is significantly improved compared to DSB modulation in Figure 9. As shown in Figure 10, the BER performance of $1.5 \mathrm{GHz}$ is the best in that the transmitted power is larger than $-8 \mathrm{dBm}$.

Figure 11 presents BER's dependence on the location of the amplifier (EDFA) on the link with the transmitted power of $-5 \mathrm{dBm}$ in the OSSB modulation case. We can observe that without ASE, the BER is almost constant when the amplifier is near the demultiplexer. However, when ASE noise is considered, the closer the amplifier is to the receiver, the worse BER is. We found that if the EDFA is placed at $1 \mathrm{~km}$ from the transmitter, BER will reach to the smallest value. The reason is that when the amplifier is far away from the receiver, the noise power will be attenuated along with the fiber link.

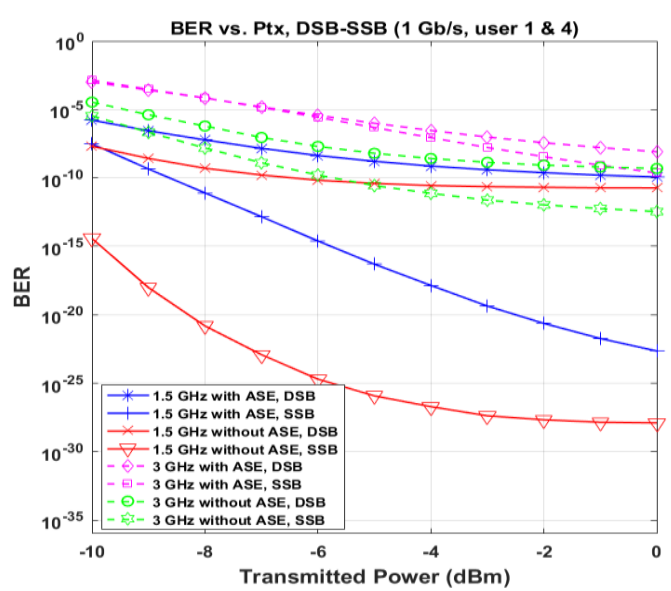

Figure 8. BER vs. transmitted power for the first and the fourth RF channels, respectively

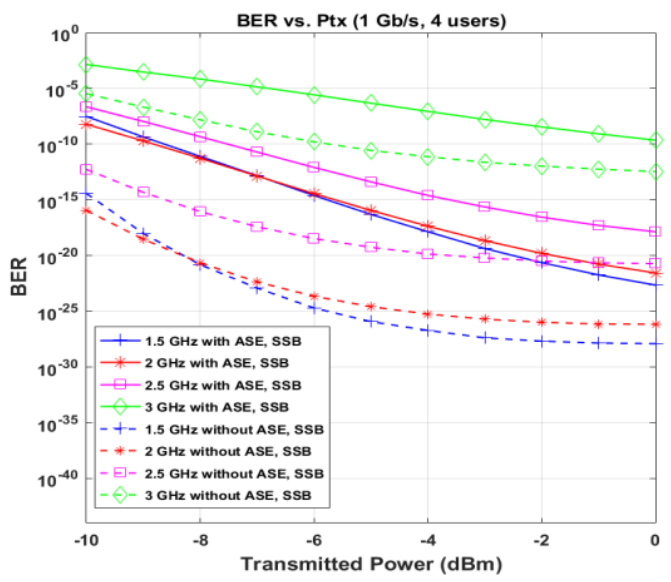

Figure 10. BER vs. transmitted power with SSB modulation

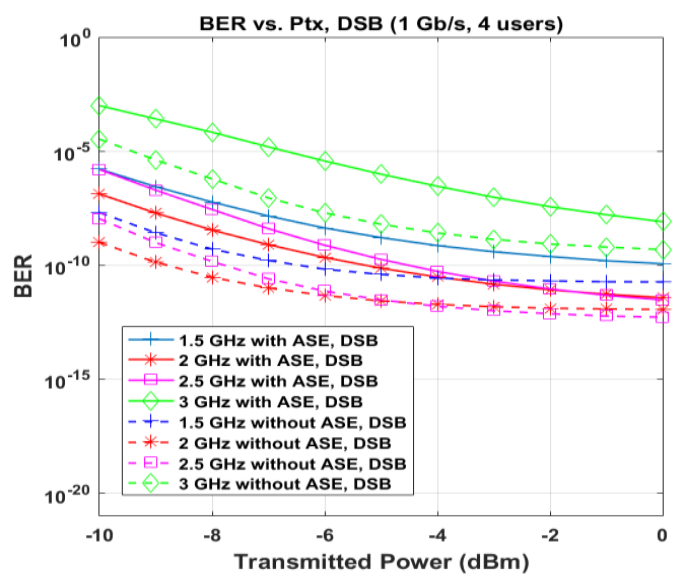

Figure 9. BER vs. transmitted power with DSB modulation

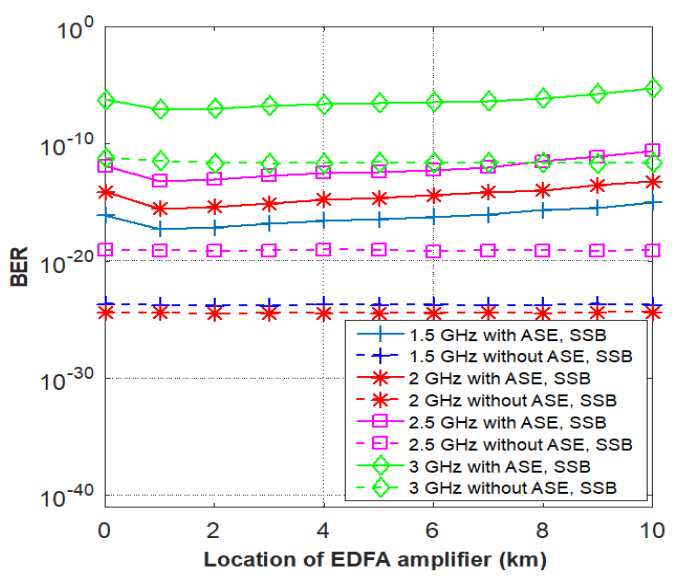

Figure 11. BER vs. location of EDFA amplifier with SSB modulation

In Figure 12, we consider the effect of FWM on WDM-RoF system performance for two cases of equal channel spacing (193.1 THz, 193.2 THz, 193.3 THz, and $193.4 \mathrm{THz}$ ) and unequal channel spacing (193.1 THz, 193.2 THz, 193.35 THz, and 193.6 THz). We investigate BER vs. transmitted power in OSSB and DSB modulation configurations. It is seen that, in unequal channel spacing, the BER of all four signal channels has been significantly improved compared to the case of equal channel spacing, especially for channels 3 and 4 . It is because the new spurious wavelengths did not appear in the original signal channels, so there was no added noise (crosstalk), as shown in section 2.2. 


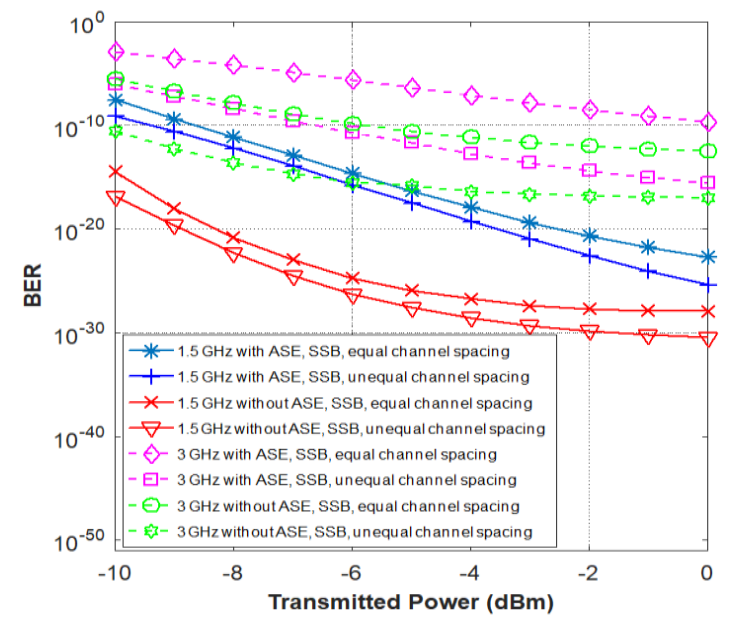

(a)

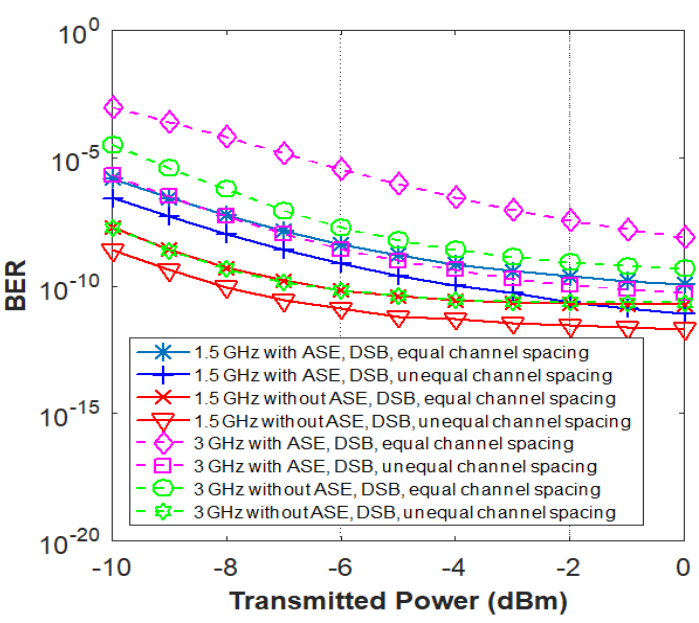

(b)

Figure 12. BER vs. transmitted power for two cases of equal and unequal channel spacing,

(a) OSSB modulation, (b) DSB modulation

Other useful information for system design can be obtained from Figure 13. we fix transmitted power $P_{\text {in }}=-5 \mathrm{dBm}$, keep the same voltage parameters for the system to operate in DSB and OSSB modes. We examine BER versus EDFA gain for cases, with and without ASE noise. It is seen that when the amplification factor increases, the BER decreases; it reaches the minimum value when gain $=20 \mathrm{~dB}$. However, when the amplification factor is greater than $20 \mathrm{~dB}$, the BER curve tends to increase rapidly. It is because as the amplification coefficient increases, the effect of the nonlinear effects will be more significant.

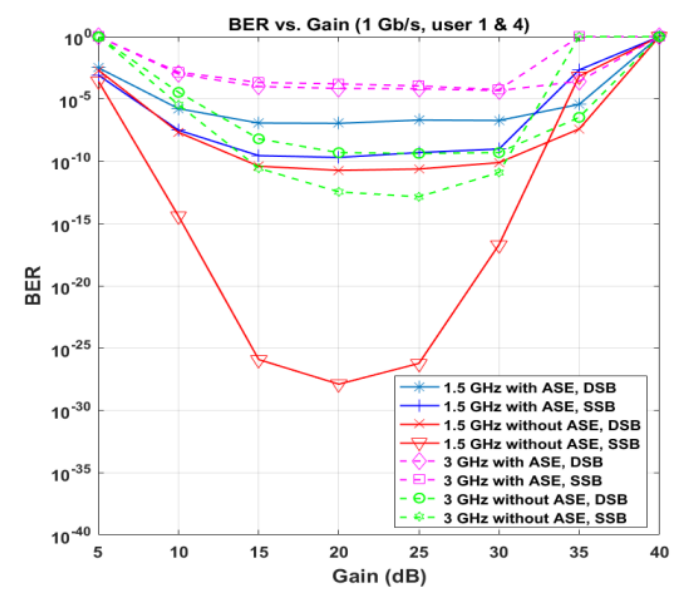

Figure 13. BER vs. EDFA gain

\section{RESULT AND DISCUSSION}

RoF is an analog transmission system. There are many ways to increase the performance of RoF. However, the use of the SCW/WDM technique has shown remarkable efficiency. It allows combining multiple RF signals, traveling on different wavelengths on the same optical fiber, exploiting the optical network's bandwidth, and increasing the system capacity [21, 22]. During the modulation process, each RF signal will produce two side-bands. If more RF signals are mixed and modulated together, it will produce higher harmonics that come in the same frequency range as another RF signal, which causes signal distortion and affects the system [14]. Therefore, the creation of a single-sideband signal is significant. Moreover, EDFA amplifiers are used in the system to compensate for transmission attenuation. Still, it also produces ASE noise that reduces the RoF system's performance, so it is necessary to investigate the effect of ASE noise. 
In this paper, we analyzed the effects of ASE noise, switching voltage, bias voltage, DSB, and SSB modulation methods on the performance of RoF-based systems. We noticed that the different modulation configurations, ASE noise, FWM, and drive voltages of the MZM modulator to play an important role in performance improvement. It reduces noise, chromatic dispersion, and noise figure, and bit error rate (BER) of the RoF systems. Simulation results present that the lowest BER was achieved by using OSSB modulation, unequal channel spacing (193.1 THz, 193.2 THz, $193.35 \mathrm{THz}$, and $193.6 \mathrm{THz}$ ) with switching voltage of $6 \mathrm{~V}=\mathrm{V} \pi$ and bias voltage of $3 \mathrm{~V}=12 \mathrm{~V} \pi$. Besides, we studied the effects of EDFA noise on the performance of SCM/WDM-based RoF. Other noise and interference, such as thermal noise, shot noise, beat noise, are considered in our model and simulation. It is seen that the presence of ASE noise significantly reduces system performance. The position of EDFA on the link between WDM Mux and WDM Demux is very important because it helps reduce ASE noises. The EDFA's best position is $1 \mathrm{~km}$ from the transmitter when the total link distance is $10 \mathrm{~km}$. The results of this paper have contributed to improving the system's performance and comparing in more detail the advantages and disadvantages of DSB and OSSB techniques. Figure out the extent of ASE noise, FWM, which will help deploy RoF systems in the future. The Table 2 below compares the results of the paper with previous works.

Table 2. Compare the results of the paper with the previous works (at $193.1 \mathrm{THz}, P_{\text {in }}=5 \mathrm{dBm}$ )

\begin{tabular}{cccc}
\hline Name & The paper & {$[19]$} & {$[20]$} \\
\hline Switching voltage & $6 \mathrm{~V}$ & $8 \mathrm{~V}$ & $4 \mathrm{~V}$ \\
Bias voltage2 & $3 \mathrm{~V}$ & not to mention & not to mention \\
Q factor & 10.9952 & 9.120 & 8 \\
BER (with ASE) & $2.013 \times 10^{-28}$ & $\sim 10^{-17}$ & $\sim 10^{-16}$ \\
\hline
\end{tabular}

\section{CONCLUSION}

In this paper, we have proposed an improved model of the WDM-RoF system using unequal channel spacing, SCM, and $1 \mathrm{~km}$ EDFA. Simulation results show that the lowest BER was archived using OSSB modulation, unequal channel spacing (193.1 THz, 193.2 THz, $193.35 \mathrm{THz}$, and $193.6 \mathrm{THz}$ ) with a switching voltage of $6 \mathrm{~V}=\mathrm{V} \pi$ and bias voltage of $3 \mathrm{~V}=12 \mathrm{~V} \pi$. We also investigate the effects of ASE noise, FWM, and the amplifier's location to bit error rate and noise figure of the system. We found that when the total link distance is $10 \mathrm{~km}$, if the EDFA is placed at $1 \mathrm{~km}$ from the transmitter, the ASE noise would be eliminated. Finally, our improved model of the RoF communication system is compared to the conventional one in terms of BER.

\section{REFERENCES}

[1] Hussein Ahmed Mahmood and Riyadh Khlf Ahmed, "Radio over fiber performance evaluation in optical communication system utilizing FBG under different DCF schemes for DPSK format," Journal of Engineering and Applied Sciences, vol. 14, no. 4, pp. 1130-1137, 2019.

[2] Sardar Irfanullah A, Gul Shaira Banu and Amjath Fareeth, "Performance prediction of 5G: The next generation of mobile communication," International Journal of Next-Generation Networks (IJNGN), vol. 9, no. 1, pp. 1-9, 2017.

[3] Kaoutar Saidi Alaoui, Foshi Jaouad, and Zouine Younes, "Radio over fiber system based on a hybrid link for next generation of optical fiber communication," International Journal of Electrical and Computer Engineering (IJECE), vol. 9, no. 4, pp. 2571-2577, Aug. 2019.

[4] Anas Ali Hussien, and Adnan Hussein Ali, "Comprehensive investigation of coherent optical OFDM-RoF employing 16QAM external modulation for long-haul optical communication system," International Journal of Electrical and Computer Engineering (IJECE), vol. 10, no. 3, pp. 2607-2616, Jun. 2020.

[5] David Wake, "Radio over fiber systems for mobile applications," in radio over fiber technologies for mobile communications networks, H. Al-Raweshidy and S. Komaki, ed., Artech House, Inc, USA, 2002.

[6] Hyoung-Jun Kim and Jong-In Song, "All optical frequency down-conversion technique utilizing a four wave mixing effect in a single semiconductor optical amplifier for wavelength division multiplexing radio-over-fiber applications," Opt. Express, vol. 20, no. 7, pp. 8047-8054, 2009.

[7] Mustafa A. Jalil, and Aied K. AL-Samarrie, "Radio-over-fibersystem capacity improvements by using wavelength division multiplexing and subcarrier multiplexing techniques," Eng. \& Technol. Journal, vol. 33, no. 6, pp. 1416-1428, 2015.

[8] Surbhi Jain and Brintha Therese A., "Four wave mixing nonlinearity effect in WDM radio over fiber system," International Journal of Scientific Engineering and Technology, vol. 4, no. 3, pp. 154-158, 2015.

[9] Shree Prakash Singh, Subrat Kar and V. K. Jain, "An approach to reduce four-wave mixing effect on existing G.653 fibers for WDM applications," 2006 IFIP International Conference on Wireless and Optical Communications Networks, Bangalore, Apr. 2006, pp. 1-5. 
[10] Haider Abd, Norashidah Md. Din, M. H. Al-Mansoori, F. Abdullah, and H. A. Fadhil, "Four-wave mixing crosstalk suppression based on the pairing combinations of differently linear-polarized optical signals," The Scientific World Journal, 2014.

[11] Kanchan Chaudhary and Kulwinder Singh Malhi, "Design and performance analysis of subcarrier multiplexed radio over fiber optical transmission system," International Journal Of Scientific \& Technology Research, vol. 8, no. 11, pp. 3720-3725, Nov. 2019.

[12] S. Revathi, G. Aarthi, "Performance analysis of wave length division and sub carrier multiplexing using different modulation techniques," International Journal of Engineering Research and Applications (IJERA), vol. 1, no. 2, pp. 317-320, 2014.

[13] Rongqing Hui, Benyuan Zhu, Renxiang Huang, Christopher T. Allen, Kenneth R. Demarest, and Douglas Richards, "Subcarrier multiplexing of highspeed optical transmission," Journal of Lightwave Technology, vol. 20, no.3, pp. 417-427, 2002.

[14] Aparna R. and S. A. Chandran, "Performance analysis of optical communication system using wavelength division and sub carrier multiplexing," International Journal of Engineering Research \& Technology (IJERT), vol. 4, no. 01, pp. 342-345, Jan. 2015.

[15] Anthony Ng' oma, Po-Tsung Shih, Jacob George, Frank Annunziata, Michael Sauer, Chun-Ting Lin, Wen-Jr Jiang, Jyehong Chen and Sien Chi, "21 Gbps OFDM wireless signal transmission at $60 \mathrm{GHz}$ using a simple IMDD radioover-fiber system," in Optical Fiber Communication Conference, OSA Technical Digest (CD) (Optical Society of America), San Diego, CA, 2010.

[16] B. Zhou, W. Nai, Y. Ge, Y. Xing and H. Zhu, "Performance Analysis on Single Sideband Modulated Radio-OverFiber System Carrying Amplitude Shift Keying Signal with Different Duty Cycle," 2018 IEEE 9th International Conference on Software Engineering and Service Science (ICSESS), Beijing, China, 2018, pp. 962-965.

[17] Ricardo Avó, Paula Laurêncio and Maria C. R. Madeiros, "Transmission performance of mm-waves on radio over fiber systems: dispersion and intermodulation issues," First IFIP WG 5.5/SOCOLNET Doctoral Conference on Computing, Electrical and Industrial Systems (DoCEIS), Costa de Caparica, Portugal, Feb. 2010, pp. 287-294.

[18] Mithun De, Indranil Mondal and Amit Kumar Das, "Studied on a multicladded Erbium doped dispersion compensating fiber amplifier," Computer Science \& Information Technology, vol. 3, no. 2, pp. 473-484, 2013.

[19] Noor J. Jihad, "The impact of switching voltage of Mach-Zehnder modulator on subcarrier multiplexing amplitude shift keying radio over fiber system," Tikrit Journal of Engineering Sciences, vol. 24, no. 3, pp. 78-83, 2017.

[20] Kamaljit Singh Bhatia, Sandeep Singh, Sakshi Sharma, Harsimrat Kaur, "Performance analysis of RoF link using Mach-Zehnder modulator and its parameters," in International Conference of Technology, Management and Social Sciences, Special Issue ICTMS-15, Dec. 2015, pp. 115-120.

[21] M. Arief, S. M. Idrus and S. Alifah, "The SCM/WDM system model for radio over fiber communication link," 2008 IEEE International RF and Microwave Conference, Kuala Lumpur, Malaysia, 2008, pp. 344-347. doi: 10.1109/RFM.2008.4897427.

[22] Arief Marwanto, Sevia M. Idrus, and Abu Sahmah Mohd. Supa'at, "Performance analysis of EDFA for SCM/WDM radio over fiber communication link," Recent Trends in Radio Over Fiber Technology. Penerbit UTM, Johor, pp. 209-212, 2008.

[23] M. T. Al-Qdah and M. S. Sharawi, "Analysis of the performance of Subcarrier Multiplexed transmission system employing OSSB modulation," 2008 5th International Multi-Conference on Systems, Signals and Devices, Amman, 2008, pp. 1-7. doi: 10.1109/SSD.2008.4632899

[24] Hilal Ahmad Sheikh and Anurag Sharma, "A radio over fiber (RoF) based single side-band modulated passive optical network (PON) using Mach Zender modulator based on different electrical phase shifts," Journal of Optical Communications, pp 1-6, 2018.

[25] Rastislav Róska, "Optical fiber and wireless communications," chapter 9, "Receiver performance improvement in radio over fiber network transmission, " Lisawati Sainawi and Khadijah Ismail, Intech, 2017.

[26] Harpreet Singh, Maninder Lal Singh, Rajandeep Singh, "A novel full duplex 16 Gbps SCM/ASK radio over fiber WDM-PON sharing wavelength for up- and down-link using bidirectional reflective filter," Optik-Int. J. Light Electron Opt., vol. 125, no. 14, pp. 3473-3475, 2014.

[27] Baljeet Kaur, Ajay K. Sharma, Vinod Kapoor, "Performance improvement on OVSB based WDM RoF-EPON link using SOA with DCF and FBG," Optik, vol. 125, no. 9, pp. 2062-2065, 2014.

[28] R. Pradeep, G. S. Karthikeya, M. Jeevan and N. Vijayakumar, "A novel architecture for full duplex WDM radio over fiber system converged with wired network using dual drive MZM and light remodulation using RSOA," 2012 Ninth International Conference on Wireless and Optical Communications Networks (WOCN), Indore, 2012, pp. 1-5. Doi: 10.1109/WOCN.2012.6331893.

[29] Wei Li, Wen Ting Wang and Ning Hua Zhu, "Photonic generation of radio frequency waveforms based on dual parallel Mach Zehnder modulator," IEEE Photonics Journal, vol. 6, no. 3, 2014.

[30] Govind P. Agrawal, "Fiber-optic communications systems," 3rd Edition., John Wiley \& Sons, Inc, 2002.

[31] Gerd Keise, "Optical fiber communications," Fourth Edition, McGraw-Hill Higher Education, 2011. 


\section{BIOGRAPHIES OF AUTHORS}
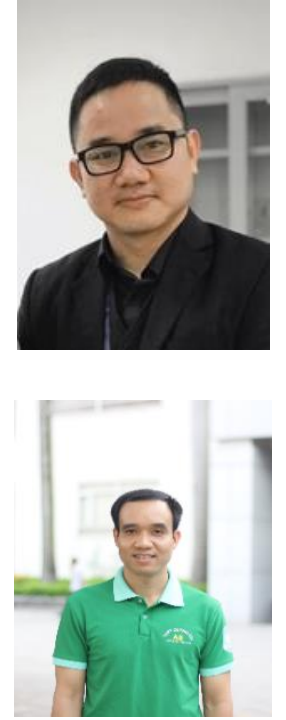

Duc-Tan Tran is an Associate professor and Vice Dean of the Faculty of Electrical and Electronic Engineering, Phenikaa University. He has published over 150 research papers. His publications received the "Best Paper Award" at the 9th International Conference on Multimedia and Ubiquitous Engineering (MUE-15) and International Conference on Green and Human Information Technology (ICGHIT-2015). He was the recipient of the award for the excellent young researcher from Vietnam National University in 2008, Hanoi, and the third prize in the contest "Vietnamese Talents" in 2008. His main research interests include the representation, processing, analysis, and communication of information embedded in signals and datasets. $\mathrm{He}$ serves as a TP Co-chair, technical committee program member, track chair, session chair, and reviewer of many international conferences and journals.

Ninh Trung Bui was born in 1981 in Thai Binh. He received his B.Sc, M.Sc, and Ph.D. Degrees, respectively in 2004, 2008, and 2017 at the University of Engineering and Technology (UET), Vietnam National University-Hanoi, Vietnam (VNUH), where he has been a lecturer since 2005. He is the author and co-author of 15 papers on networks and communications. His present research interest is in networks and optical communication. 